\title{
Intervalos de Confianza para el coeficiente alfa de Cronbach: aportes a la investigación pediátrica
}

\author{
Confidence Intervals for Cronbach's \\ alpha coefficient: contributions to \\ pediatric research.
}

En la actualidad, es cada vez más común encontrar estudios que brinden evidencias de la confiabilidad de las puntuaciones obtenidas, por instrumentos de medida utilizadas en investigaciones en ciencias de la salud. ${ }^{1}$ Este es el caso de la valiosa contribución de Aguilar-Navarro et al, ${ }^{2}$ quienes adaptaron la Escala de Actitudes hacia la Alimentación Infantil de lowa (IIFAS) en México, reportando la confiabilidad a través del alfa de Cronbach.

El coeficiente alfa de Cronbach es el más utilizado para la estimación de la confiabilidad bajo el método de consistencia interna ${ }^{3,4}$ y expresa qué porcentaje de varianza observada es atribuida a la varianza verdadera y que porcentaje a la varianza del error de medida. ${ }^{5}$ Los resultados del estudio de Aguilar-Navarro et al., ${ }^{2}$ indican que la dimensión juicio a favor de la lactancia materna $(\alpha=0.71)$ se encuentra al límite de lo mínimamente aceptable; mientras que los valores de alfa de la escala total $(\alpha=0.65)$ y de las dimensiones juicios a favor de fórmulas lácteas ( $\alpha=0.63)$ y juicios con componente psicosocial y de salud ( $\alpha=0.49$ ) están por debajo de ese límite. Si bien los autores señalan que la IIFAS permite obtener puntuaciones confiables, esta conclusión puede no ser la correcta.

La magnitud del alfa de Cronbach puede sufrir variaciones producto de la influencia del error de medida, lo que hace necesario calcular sus intervalos de confianza (IC). En este sentido, la presente carta tiene como objetivo complementar los valores del alfa de Cronbach del estudio de Aguilar-Navarro et al., ${ }^{2}$ con el reporte de los intervalos de confianza. El cálculo de los intervalos de confianza es un procedimiento recomendado desde hace algunos años ${ }^{6}$ que permiten una estimación de todos los posibles valores poblacionales del coeficiente alfa de acuerdo a un determinado nivel de confianza. ${ }^{7}$ Los intervalos de confianza pueden estimarse haciendo uso del módulo IC Alfa ${ }^{7}$ desarrollado en base al método de Fisher. ${ }^{8}$ La interpretación de los intervalos de confianza es sencilla, donde un límite inferior igual o mayor a 0.70 brindaría evidencia de una confiabilidad aceptable. ${ }^{7}$ Debido a lo breve de la carta, y para un mayor conocimiento acerca de las fórmulas matemáticas utilizadas para el cálculo de los intervalos de confianza, se recomienda la lectura de la literatura metodológica especializada.9,10 
Un re-análisis de los datos de consistencia interna presentes en el cuadro 4 del estudio de Aguilar-Navarro et al., ${ }^{2}$ permiten concluir que la escala total ( $\mathrm{IC}=0.588,0.704)$, así como las dimensiones juicio a favor de la lactancia materna (IC $=0.657,0.756$ ), juicios a favor de fórmulas lácteas $(\mathrm{IC}=0.566,0.687)$ y juicios con componente psicosocial y de salud $(\mathrm{IC}=0.410$, 0.562) presentan límites inferiores de intervalos de confianza muy por debajo de lo mínimo requerido. Lo anterior, permite concluir que existe demasiado error de medida en las puntuaciones del IIFAS, lo que podría afectar los análisis estadísticos posteriores y las conclusiones de aquellos estudios que utilizaran esta escala.

Es así, que se sugiere el cálculo de los intervalos de confianza en investigaciones que empleen instrumentos de medida, similares al IIFAS, más aún si sus resultados van a ser utilizados en la toma de decisiones en el ámbito pediátrico.

\section{REFERENCIAS}

1. Campo-Arias A, Oviedo HC. Propiedades psicométricas de una escala: la consistencia interna. Rev Salud Pública. 2008; 10(5): 831-839.

2. Aguilar-Navarro HJ, Coronado-Castilleja A, Gómez-Hernández $\mathrm{OJ}$, Cobos-Aguilar $\mathrm{H}$. Adaptación de lalowa Infant
Feeding Attitude Scale en poblaciónmexicana. Acta Pediatr Mex. 2016;37(3):149-158.

3. Cronbach LJ. My current thoughts on coefficient Alpha and successor procedures. Educ Psychol Meas. 2004; 64(3): 391-418.

4. Gadermann AM, Guhn M, Zumbo B D. Estimating ordinal reliability for Likert-type and ordinal item response data: A conceptual, empirical, and practical guide. Pract Assess Res Eval. 2012; 17(3): 1-13.

5. Domínguez-Lara S.A. Secretos del coeficiente alfa. Actas Urol Esp. 2016; 40(7): 471.

6. Fan X, Thompson B. Confidence intervals about score reliability coefficients, please: an EPM guide lines editorial. Educ Psychol Meas. 2001; 61: 517-531.

7. Domínguez-Lara SA, Merino-Soto M. ¿Por qué es importante reportar los intervalos de confianza del coeficiente alfa de Cronbach? Revista Latinoamericana de Ciencias Sociales, Niñez y Juventud. 2015; 13(2): 1326-1328.

8. Fisher R. Statistical methods for research workers. Edinburgh: Oliver \&Boyd; 1950.

9. Romano JL, Kromrey JD, Hibbard ST. A Monte Carlo study of eight confidence interval methods for coefficient alpha. Educ Psychol Meas. 2010; 70(3): 376-393.

10. 1Romano JL, Kromrey JD, Owens CM, Scott HM. Confidence interval methods for coefficient alpha on the basis of discrete, ordinal response items: Whichone, ifany, is the best? The Journal of Experimental Education. 2011; 79(4): 382-403.

Tomás Caycho-Rodríguez Universidad Privada del Norte Av. Tingo María 1122, Breña, Lima tomas.caycho@upn.pe Teléfono: +511941715057 